\title{
Primary Processes in Radiation-Induced Crosslinking of Poly(2-phenylbutadiene)
}

\author{
Dedicated to the Memory of the late Professor Ichiro Sakurada \\ Hitoshi YAmaOKA, Kazuo Kato, ${ }^{*}$ and Seizo OKAMURA* \\ Research Reactor Institute, Kyoto University, \\ Kumatori, Osaka 590-04, Japan \\ * Department of Polymer Chemistry, Kyoto University, \\ Sakyo-ku, Kyoto 606, Japan \\ (Received February 9, 1987)
}

\begin{abstract}
The radiation-induced crosslinking of poly(2-phenylbutadiene) (PPB) in ethylene dichloride solution was studied in vacuum at $303 \mathrm{~K}$. The $G$ value of the crosslinking was estimated to be about 7.2. In order to detect the reaction intermediates under irradiation, optical absorption spectra in rigid matrices and ESR spectra in bulk were measured. The absorption spectra due to radical cation of PPB and due to $\alpha, \alpha$-disubstituted benzyl cation were observed in butyl chloride glass. ESR spectra owing to polyenyl type radical was found in the irradiated specimens of PPB and Diels-Alder type dimer of 2-phenylbutadiene. The primary processes in radiation-induced crosslinking of PPB were discussed on the basis of the results obtained.

KEY WORDS Poly(2-phenylbutadiene) / Radiation Effect / Crosslinking /

Primary Process / $G$ Value /
\end{abstract}

The radiation chemistry on diene polymers have been extensively investigated in recent years. ${ }^{1,2}$ A large number of studies on transient species produced in the polymers during irradiation have provided us with the fundamental information on elucidating the mechanism of radiation-induced reactions. However, these studies have almost limited to the conventional diene polymers such as polybutadiene and polyisoprene.

In a previous communication, ${ }^{3}$ we reported that the crosslinking of poly(2-phenylbutadiene) (PPB) occurred under irradiation in vacuum. The purposes of the present paper are to study the details of the crosslinking reaction and to detect the transient intermediates in $\gamma$-irradiated PPB, being devoted to clarify the primary processes of the crosslinking.

\section{EXPERIMENTAL}

\section{Materials}

2-Phenylbutadiene was synthesized by the dehydration of the corresponding alcohol obtained by the Prins reaction of $\alpha$-methylstyrene or by the Grignard reaction of acetophenone. PPB with high content of 1,4-structural units was prepared by an anionic polymerization with $n$-butyllithium. The microstructure of the polymer was determined by applying Ambrose's method ${ }^{4}$ and was found to be $88 \%$ of 1,4-units, $6 \%$ of 1,2 -units, and $6 \%$ of $3,4-$ units. Diels-Alder type dimer of 2-phenylbutadiene, which is formed during the storage of the monomer at low temperature, was purified by repeated recrystallization from methanol. Ethylene dichloride (EDC), sec-butyl chloride $(\mathrm{BuCl})$, and bromobenzene $(\mathrm{PhBr})$ were distilled over calcium hydride before use. 


\section{Irradiation and Measurements}

All samples were degassed by several freezethaw cycles under high vacuum and then sealed off. Irradiation of the samples was performed with a $12000 \mathrm{Ci}{ }^{60} \mathrm{Co} \gamma$-ray source at the Research Reactor Institute, Kyoto University.

Experiments on the crosslinking of PPB were carried out at $303 \mathrm{~K}$. The polymer concentration in EDC solution was $1.2 \mathrm{~g} \mathrm{dl}^{-1}$ $\left(9.2 \times 10^{-2} \mathrm{M}\right.$ based on the monomer unit). The apparatus used for evaluation of the crosslinking was composed of a cylindrical quartz tube with an attached capillary viscometer. After irradiation, the solution was poured into a large excess of methanol and precipitated polymer was recovered by filtration. Extraction of sol fraction in the irradiated polymer was made for $20 \mathrm{~h}$ in a conventional Soxhlet apparatus using toluene as a solvent.

Optical absorption measurements in rigid matrices were carried out with quartz cell of $2 \mathrm{~mm}$ path length at $77 \mathrm{~K}$ by using a Shimadzu MPS-50L spectrometer. Photobleaching of the irradiated samples was achieved with a tungsten lamp. ESR spectra were measured with a Varian E-3 X-band spectrometer at $77 \mathrm{~K}$ by employing $100 \mathrm{kHz}$ modulation at a microwave power of $1 \mathrm{~mW}$.

\section{RESULTS}

\section{Crosslinking of $P P B$}

The change in viscosity of PPB solution is represented in Figure 1 as a function of dosage, where $\left(\eta_{\mathrm{sp}} / c\right)_{0}$ is the viscosity number of PPB solution before irradiation and $\left(\eta_{\mathrm{sp}} / c\right)_{\mathrm{D}}$ is that at irradiation dose $D$. The viscosity of the polymer solution increased with an increase in the absorbed dose, indicating the occurrence of crosslinking. With further irradiation, the gel formation of PPB was observed as shown in Table $\mathrm{I}$. The point at which an insoluble network first starts to form is called the gel point and the critical irradiation dosage at the gel point is termed the gelation dose $\left(\gamma_{\mathrm{gel}}\right)$.

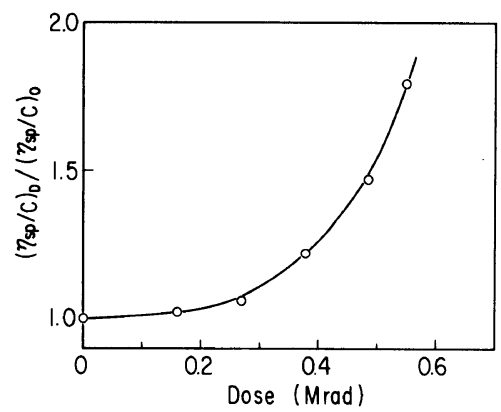

Figure 1. Ratio of $\left(\eta_{\mathrm{sp}} / C\right)_{\mathrm{D}} /\left(\eta_{\mathrm{sp}} / C\right)_{0}$ for irradiated PPB in EDC as a function of dose. Dose rate, $0.22 \mathrm{Mrad} \mathrm{h}^{-1}$; temp, $303 \mathrm{~K}$.

Table I. Relation between gel fraction of irradiated PPB and dose ${ }^{a}$

\begin{tabular}{|c|c|c|}
\hline \multirow{2}{*}{ Exptl. No. } & Dose & Gel fraction \\
\hline & Mrad & $\%$ \\
\hline 1 & 0.43 & 0 \\
\hline 2 & 0.76 & 2 \\
\hline 3 & 0.86 & 11 \\
\hline 4 & 1.08 & 30 \\
\hline 5 & 1.30 & 51 \\
\hline
\end{tabular}

a Dose rate, $0.22 \mathrm{Mrad} \mathrm{h}^{-1}$; temp, $303 \mathrm{~K}$.

From the plots of experimental results in the form of $\log$ (sol fraction) against irradiation dosage, the value of $\gamma_{\text {gel }}$ is generally determined by extraporation of the sol-dosage curve to $\log ($ sol fraction $)=1$. By applying this method to the results of Table I, the $\gamma_{\text {gel }}$ value in the present system was obtained to be about 0.74 Mrad.

Since the chain scission due to radiolysis in PPB under vacuum is estimated to be essentially zero, the $G$ value for crosslinking, $G(X)$, can be given by the following equation:

$$
G(X)=0.48 \times 10^{6} / \gamma_{\text {gel }} \times \bar{M}_{w}
$$

where $\gamma_{\text {gel }}$ is expressed as the unit of Mrad and $\bar{M}_{w}$ is the weight average molecular weight of the polymer before irradiation. ${ }^{5}$ Although the relation between the viscosity and the molecular weight for PPB has not been known, $\bar{M}_{w}$ was roughly estimated to be $9 \times 10^{4}$ by using 
the viscosity equation for the copolymer of ethylene with $\alpha$-methylstyrene. ${ }^{6}$ Substituting the above values for $\gamma_{g e l}$ and $\bar{M}_{w}$ in equation 1 , the $G(X)$ in the present system was obtained to be about 7.2.

\section{Optical and ESR Measurements}

A matrix of $\mathrm{BuCl}$ is often used to stabilize positive ions. The positive holes in the matrix upon irradiation migrate in the glassy matrix and form radical cations of solute molecules. Diels-Alder type dimer of 2-phenylbutadiene (I), which has a double bond conjugated with a phenyl group, was used as a model compound of 1,4-structural unit of PPB.<smiles>C=CC12CC=CC(CC1)C(c1ccccc1)CC2</smiles>

The absorption spectra of $\gamma$-irradiated $\mathrm{BuCl}$ glasses containing $0.05 \mathrm{M}$ and $0.4 \mathrm{M}$ of the dimer at $77 \mathrm{~K}$ are shown in Figures 2(a) and (b), respectively. Three absorption bands at 370,400 , and $680 \mathrm{~nm}$ were observed in both spectra and assigned to the radical cation of (I). In the case of higher concentration of the dimer, the spectrum shows an additional band at about $470 \mathrm{~nm}$. From an analogous consideration with $\gamma$-irradiated $\alpha$-methylstyrene by

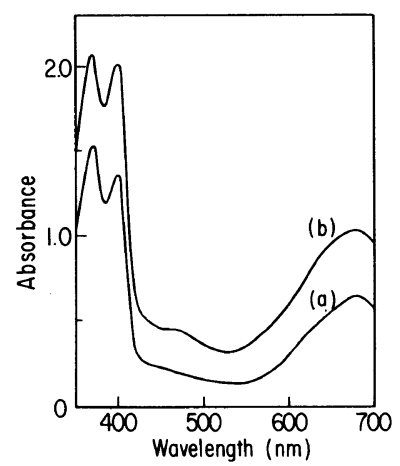

Figure 2. Abșorption spectra of irradiated 2-phenylbutadiene dimer in $\mathrm{BuCl}$ matrix at $77 \mathrm{~K}$. Dimer concn: (a) $0.05 \mathrm{M}$; (b) $0.4 \mathrm{M}$; total dose, $0.45 \mathrm{Mrad}$.
Irie et al., ${ }^{7}$ the absorption band at $470 \mathrm{~nm}$ was assigned to the sandwich-type dimer radical cation (II) proposed by Badger and Brocklehurst. $^{8}$

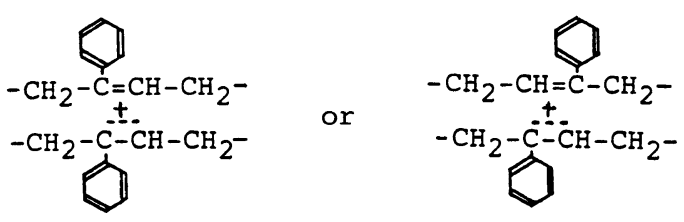

The absorption spectrum of irradiated PPB in $\mathrm{BuCl}$ matrix are shown in Figure 3(a). The spectrum was rather vague and the peaks of species seemed to overlap with the bands due to the solvent cation at 380 and $540 \mathrm{~nm} .{ }^{9}$ In the case of PPB, therefore, the absorption spectra were taken after photobleaching with a visible light. The photobleaching process facilitates the positive charge transfer from the solvent cation to the polymer, which weakens the absorption bands due to the solvent cation and enhances those of the polymer radical cation. The resulted spectrum (Figure 3(b)) has four absorption maxima at $380,400,465$, and $650 \mathrm{~nm}$. On the basis of the assignment for the irradiated dimer, three bands at 380, 400, and $650 \mathrm{~nm}$ are attributable to the radical cation of PPB and the band at $465 \mathrm{~nm}$ is due to the sandwich-type dimer radical cation. The absorption band at $400 \mathrm{~nm}$ can be assigned to the

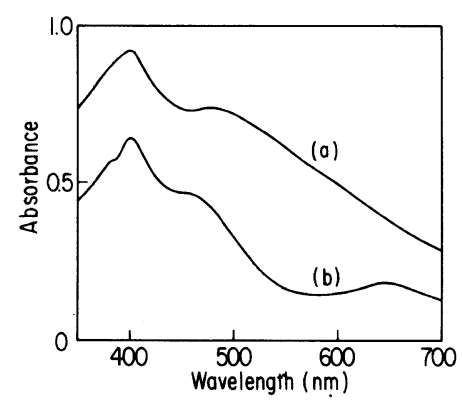

Figure 3. Absorption spectra of irradiated PPB in $\mathrm{BuCl}$ matrix at $77 \mathrm{~K}$. (a) before photobleaching; (b) after photobleaching; PPB concn, $0.09 \mathrm{M}$, total dose, 2.70 Mrad. 
$\alpha, \alpha$-disubstituted benzyl cation of the polymer (III), because this cation is known to show an absorption peak at about $400 \mathrm{~nm} .^{10,11}$

$$
-\mathrm{CH}_{2}-\stackrel{+}{\mathrm{C}}-\mathrm{CH}_{2}-\mathrm{CH}_{2}-
$$

The spectrum of $\gamma$-irradiated $\mathrm{BuCl}$ containing higher concentration of PPB $(0.36 \mathrm{M})$ is represented in Figure 4(a). Two new absorption bands at 510 and $550 \mathrm{~nm}$ were observed in addition to the four bands in Figure 3(b). These two bands were also observed in the absorption spectrum of irradiated PPB in $\mathrm{PhBr}$ glass at $77 \mathrm{~K}$ (Figure 4(b)). By $\gamma$-irradiation of $\mathrm{PhBr}$ matrix, a phenyl radical is formed through a dissociative electron capture process and a hydrogen abstraction from a solute molecule by the resulted phenyl radical subsequently occurs even at $77 \mathrm{~K} .{ }^{12}$ In the case of PPB as a solute, hydrogen atoms on the 4th carbon atom of the monomer unit would be susceptible to the hydrogen abstraction, because a resulted radical (IV) (phenylsubstituted allyl radical) is resonance stabilized and hence is reasonably stable at $77 \mathrm{~K}$. Therefore, we tentatively assigned that the absorption bands at 510 and $550 \mathrm{~nm}$ are due to the radical (IV). Bodily and Dole ${ }^{13}$ reported in the study of irradiated polyethylene that several polyenyl radicals of the structure, $-\dot{\mathrm{C}} \mathrm{H}-$ $(\mathrm{CH}=\mathrm{CH})_{n}^{-}$, show the absorption bands at

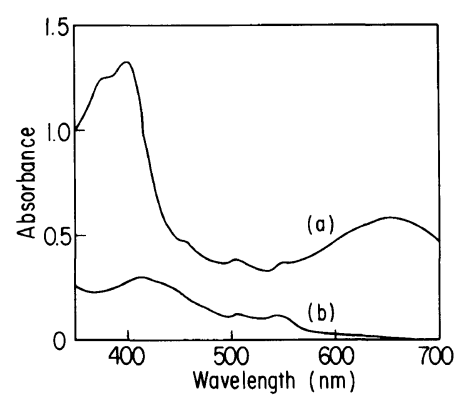

Figure 4. Absorption spectra of irradiated PPB in $\mathrm{BuCl}$ and $\mathrm{PhBr}$ matrices after photobleaching at $77 \mathrm{~K}$. (a) $0.36 \mathrm{M} \mathrm{PPB}$ in $\mathrm{BuCl}$; total dose, $0.67 \mathrm{Mrad}$. (b) 0.20 M PPB in $\mathrm{PhBr}$; total dose, 1.35 Mrad. different wavelengths and the absorption maxima uniformly shift to longer wavelengths with increasing the number of conjugated double bonds. These results seem to support our assignment of 510 and $550 \mathrm{~nm}$ bands to the radical (IV).

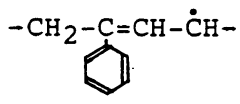

Figures 5(a) and (b) show ESR spectra of $\gamma$-irradiated PPB and Diels-Alder type dimer, respectively. The spectra are singlet with no resolved hyperfine structure. Photobleaching procedures gave no effect either on the shape or on the intensity of these spectra. On warming to room temperature, these spectra began to decay and immediately disappeared by admitting air in the system. Similar singlet spectra were observed in irradiated polyisoprene as well as polybutadiene by many researchers and were assigned to allyl or conjugated polyenyl radical. ${ }^{14}$ The results of ESR measurements provide further evidence on the presence of allylic type radicals in the present system, although an ESR spectrum of the radical (IV) has not yet been reported.

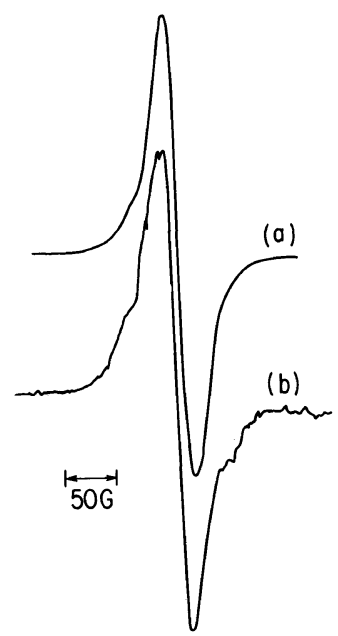

Figure 5. ESR spectra of irradiated dimer and PPB at $77 \mathrm{~K}$. (a) 2-phenylbuțadiene dimer; (b) PPB; total dose, $5.13 \mathrm{Mrad}$. 


\section{DISCUSSION}

For irradiation of bulk elastomers in vacuum at room temperature, comparatively small $G(X)$ values ranging from 0.9 for natural rubber to 3-4 for polybutadiene were reported. ${ }^{2}$ The $G(X)$ value for synthetic high cis1,4-polyisoprene was also obtained to be approximately equal to that for natural rubber. In these elastomers, the reaction mechanism of crosslinking has been established to proceed via the coupling of allylic free radicals formed during irradiation or the direct addition of free radicals to the double bonds in the main chain. In copolymers of butadiene and styrene, however, the introduction of styrene unit into butadiene chain leaded to a remarkable reduction in the $G(X)$ value and this effect was attributed to the energy transfer from butadiene chain to styrene unit of greater radiation stability. ${ }^{15,16}$

In the present study, the $G(X)$ value for PPB in EDC was estimated to be about 7.2. The crosslinking due to radical reactions would occur in irradiated PPB, because the presence of allylic type radicals were detected at $77 \mathrm{~K}$ by optical and ESR measurements. However, the radical mechanism is not sufficient to explain the higher $G(X)$ value in the case of PPB containing an aromatic ring in the monomer unit.

One possible explanation for the present system is the contribution of ionic species. In contrast to the information available on the radical reaction, only a little is known about the ionic mechanism on the crosslinking of elastomers. Heusinger and his coworkers found that the radiation induced crosslinking and cyclization in 1,2- and 3,4-polyisoprenes and 1,2-polybutadiene are initiated by carbonium ions produced by inter- and intramolecular reactions of radical ions with double bonds. ${ }^{17-19}$ Recently, Basheer and Dole reported that the contribution of ionic reactions to crosslinking in irradiated styrenebutadiene copolymers is about $25-35 \%$ of the total crosslinking yield. ${ }^{20}$

A similar situation in ionic reactions would also occur in the present system. On the assumption that the same intermediates observed in optical and ESR measurements at $77 \mathrm{~K}$ are formed in the polymer solution at room temperature, the following scheme on the primary processes of irradiated PPB can be proposed:
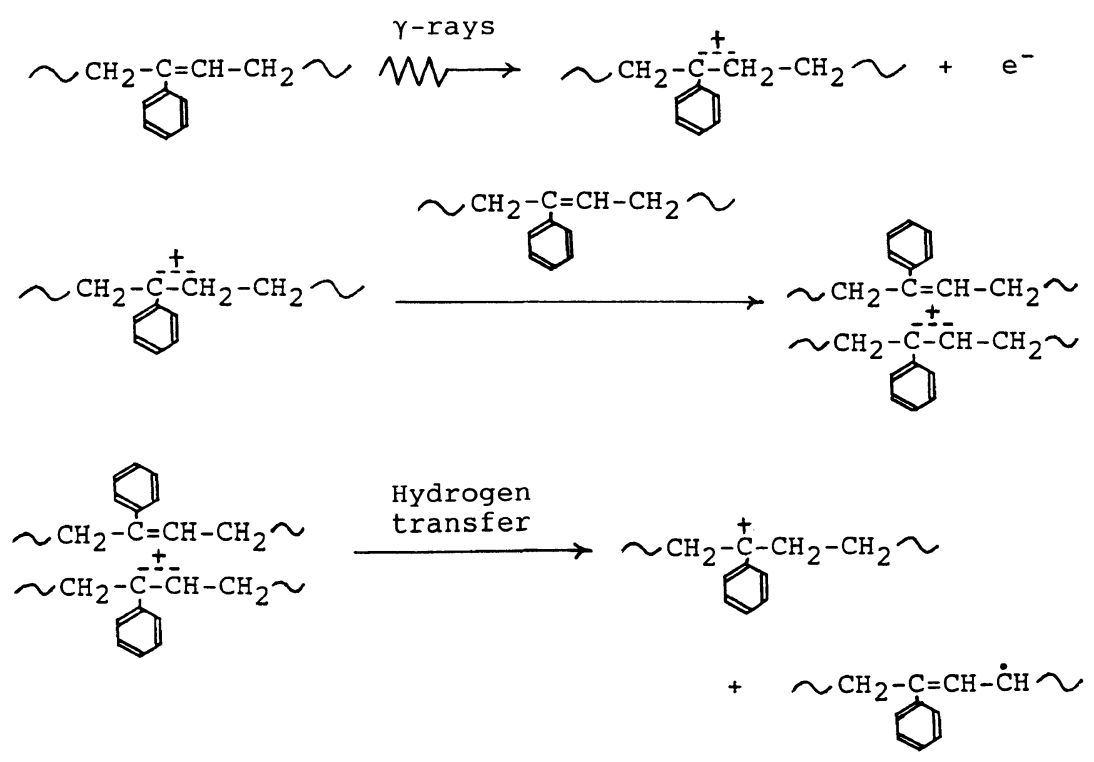
The radical cations which escape from geminate recombination resulted from reaction (a) and the carbonium ion from reaction (c) will start the crosslinking through ionmolecule reactions with double bonds of other PPB chains. The presence of EDC as a solvent would facilitate the solvation and the stability of cationic species. The progress of crosslinking should reduce their flexibility and result in the increase of the sandwich-type dimer radical cation sites. Thus the crosslinking reactions of both cationic and radical mechanisms seem to proceed simultaneously in the present system. However, the extent of ionic contribution on the crosslinking still remains unsolved.

In conclusion, a definite proof of ionic mechanism in radiation induced crosslinking of diene polymers was obtained by using PPB as a sample. Such information would be great help in understanding the characteristics of polymer radiolysis.

Acknowledgment. The authors wish to thank Dr. T. Matsuyama of Kyoto University for his collaboration in optical and ESR measurements.

\section{REFERENCES}

1. G. G. A. Bohm, "The Radiation Chemistry of Macromolecules," M. Dole, Ed., Academic Press, New York, N.Y., 1973, Vol. 2, p 195.
2. G. G. A. Bohm, Rubber Chem. Technol., 55, 575 (1982).

3. K. Kato, S. Okamura, and H. Yamaoka, J. Polym. Sci., Polym. Lett. Ed., 14, 221 (1976).

4. R. J. Ambrose and W. L. Hergenrother, Macromolecules, 5, 275 (1972).

5. A. Charlesby, "Atomic Radiation and Polymers," Pergamon Press, London, 1960, Chapter 9.

6. H. Tanzawa, T. Tanaka, and A. Soda, J. Polym. Sci., A-2, 7, 929 (1969).

7. M. Irie, H. Masuhara, K. Hayashi, and N. Mataga, J. Phys. Chem., 78, 341 (1974).

8. B. Badger and B. Brocklehurst, Trans. Faraday Soc., 65, 2582 (1969).

9. T. Shida and W. H. Hamill, J. Chem. Phys., 44, 4372 (1966).

10. N. C. Deno, J. J. Jaruzelski, and A. Schriesheim, J. Org. Chem., 19, 155 (1954).

11. J. A. Grace and M. C. R. Symons, J. Chem. Soc., 958 (1959).

12. S. Noda, K. Fueki, and Z. Kuri, Bull. Chem. Soc. Jpn, 41, 2882 (1968).

13. D. M. Bodily and M. Dole, J. Chem. Phys., 45, 1428 (1966).

14. B. Ranby and J. F. Rabek, "ESR Spectroscopy in Polymer Research," Springer-Verlag, Berlin, 1977, p 198.

15. E. Witt, J. Polym. Sci., 41, 507 (1959).

16. R. Basheer and M. Dole, Makromol. Chem., 183, 2141 (1982).

17. H. Katzer and H. Heusinger, Makromol. Chem., 163, 224 (1973).

18. A. von Raven and H. Heusinger, J. Polym. Sci., Polym. Chem. Ed., 12, 2255 (1974).

19. R. Kaufmann and H. Heusinger, Makromol. Chem., 177, 871 (1976).

20. R. Basheer and M. Dole, J. Polym. Sci., Polym. Phys. Ed., 22, 1313 (1984). 